\title{
Does antiretroviral therapy interfere with the treatment of Trichomonas vaginalis among HIV+ women?
}

\author{
Patricia Kissinger, Ph.D. ${ }^{1}$, Alys Adamski, MPH ${ }^{1}$, Rebecca A. Clark, MD, Ph.D. ${ }^{2}$, Leandro \\ Mena, MD, MPH $^{3}$, Judy Levison, MD $^{4}$, and David H. Martin, MD $^{2}$ \\ ${ }^{1}$ Tulane University School of Public Health and Tropical Medicine, Department of Epidemiology \\ 2Louisiana State University, Health Sciences Center - Department of Medicine, Section of \\ Infectious Diseases \\ ${ }^{3}$ University of Mississippi Medical Center - Department of Medicine \\ ${ }^{4}$ Baylor College of Medicine
}

\begin{abstract}
High repeat infection rates ( $8 \%$ to $20 \%{ }^{1-6}$ ) among women receiving $2 \mathrm{~g}$ dose of metronidazole (MTZ) for the treatment of Trichomonas vaginalis (TV) have brought into question the effectiveness of single dose treatment. Repeat infection rates appear to be particularly high among HIV+ women ranging from $18.3 \%$ to $36.9 \%,{ }^{278}$, suggesting that some factors specific to HIV+ women may be interfering with single dose MTZ therapy.
\end{abstract}

In our multi-centered randomized trial among HIV+ women attending two southern United States HIV outpatient clinics, we found that the 7 day $500 \mathrm{mg}$ twice daily MTZ regimen was superior to the $2 \mathrm{~g}$ single dose regimen for TV infection. ${ }^{9}$ In a subsequent stratified analysis, we discovered that superiority of multi-dose over single-dose was restricted to women with BV suggesting that this condition interfered in some way with single dose MTZ therapy. ${ }^{10}$

In this issue of the journal, Balkus et al report their observations of TV treatment outcomes in a cohort of HIV- and HIV+ female sex workers in Kenya. They found that the $2 \mathrm{~g}$ single dose MTZ treatment success rate among HIV+ women, was significantly lower for those with bacterial vaginosis (BV), corroborating our findings. However, they also found that in addition to lower treatment success rates for women with BV, that women who were receiving antiretroviral therapy (ART) also had lower treatment success.

To compare our finding to that of Balkus et al., we performed a secondary analysis of TV treatment outcomes for women randomized to the $2 \mathrm{~g}$ MTZ arm in our published study. ART and BV status were determined at baseline. ART was elicited by abstracting prescribed medication from the medical records and then confirming with the patient's self-report of whether or not they were taking the medicine. Patients' were asked which HIV medications they used by a trained study staff person using a chart with pictures of medications available during the study time period (May, 2006 to July 2009). When ART status was discrepant between medical records and patient-report, the patient-report was used. BV was diagnosed in women who had a Nugent score of > 7. Women were tested for TV using InPouch culture

Address all correspondence to: Patricia Kissinger, Ph.D. Tulane University School of Public Health and Tropic Medicine Department of Epidemiology SL-18 1440 Canal Street New Orleans, LA 70012 kissing@tulane.edu.

This is a PDF file of an unedited manuscript that has been accepted for publication. As a service to our customers we are providing this early version of the manuscript. The manuscript will undergo copyediting, typesetting, and review of the resulting proof before it is published in its final citable form. Please note that during the production process errors may be discovered which could affect the content, and all legal disclaimers that apply to the journal pertain. 
at both baseline and at the 7 day post treatment test of cure visit (TOC) visit. Treatment was administered and directly observed at the clinic and all women were offered $2 \mathrm{~g}$ MTZ treatment to deliver to their sex partner(s).

Of the 130 women in this arm, $113(86.9 \%)$ returned for their TOC visit and had complete data for Nugent score, CD4 cell count, and ART and were included in the analyses. At baseline, $54.9 \%$ of the women had BV per Nugent score, CD4 counts were as follows : < $200 / \mathrm{mm}^{3}(35.7 \%), 200-499 / \mathrm{mm}^{3}(53.1 \%),>500 / \mathrm{mm}^{3}(21.2 \%)$. Viral loads were as follows: $<50$ copies $/ \mathrm{mL}$ (43.8\%), 50-9,999 copies/mL (21.4\%), > 10,000 copies/mL (34.8\%). Of the $76(67.3 \%)$ women receiving ART, the classes of drugs as documented on the medical record and confirmed by the participant, were the following: NRTI (94.8\%), NNRTI (31.1\%) PI (58.1\%), and other (2.0\%). As a measure of ART adherence, we asked "did you take this medication as prescribed the day before" and $94.7 \%$ said yes to this question for each ART they were taking. Unlike the Balkus cohort, where all who were taking ART were receiving nevirapine-containing therapies, only 1 of our participants received nevirapine.

At TOC, $18.6 \%$ of those included in the analysis were TV positive via culture. Those receiving ART were as likely as those not receiving ART to have re-initiated sex (24.3\% vs. $14.5 \%$, P-value $=0.20$ ). Women who were receiving ART were more likely to have CD4 cell count $<200 / \mathrm{mm}^{3}(32.9 \%$ vs. $10.8 \%, \mathrm{P}=0.008)$ and viral loads $<50$ copies $/ \mathrm{mL}$ copies (54.7\% vs. $21.6 \%, \mathrm{P}=0.002$ ) compared to women not receiving ART. These two factors were similar by BV status. In multivariable logistic regression, after adjusting for the presence of BV and CD4 cell count, women who were receiving ART were 4.58 times more likely to be TV+ at TOC than women not receiving ART (1.09-19.36, P-value=0.038).

After removing the 4 women who did not adhere to their ART regimen the day before from the analysis, the effect measures on ART did not change but had a slightly narrower confidence interval (O.R. 4.72, 95\% C.I. 1.12 - 19.82, P-value 0.034).

To examine if the association between ART and TV treatment failure could be explained by $\mathrm{BV}$, a stratified analysis was performed. The association between ART and TV+ at TOC by BV status had a Cochrane's chi-square of $3.69(\mathrm{P}$-value $=0.06)$ demonstrating that after adjusting for BV, ART was associated with treatment failure. The Breslow-Day chi-square was 0.77 (P-value 0.38) indicating no interaction between BV and ART thus supporting an independent association of ART on the repeat infection rate.

There were some differences between these two studies and our analyses address several of the limitations identified by Balkus et al. in their study. We used culture rather than the less sensitive wet prep for TV detectible and therefore likely included more asymptomatic women. Future studies should use more sensitive nucleic acid amplification techniques. Follow-up assessment in our study was 7 days post completion of treatment compared to 1 month post treatment in the Balkus study. Nearly $50 \%$ of Balkus cohort had sexual exposure compared to $20 \%$ in our cohort. This difference in sexual exposure is likely attributed to differences in the cohorts (Balkus cohort were sex workers) and a longer follow-up time between baseline and TOC visit (mean of 33 days vs. 10 days). Our study, therefore, was able to reduce, but not eliminate the confounding effect of sexual exposure. Since sexual exposure was similar by ART status in our study, we think confounding was minimal. Finally, a number of ARTs were used in our cohort compared to the Balkus cohort, which was exclusively nevirapine-containing therapies, demonstrating that the ART effect is not a phenomenon specific to nevirapine.

While our study addresses some of the limitations of the Balkus et al study, neither study can establish causality since they both lacked experimentation. It is possible that ART may be a marker for some other factor that interferes with single dose MTZ treatment of TV. To 
better understand the mechanism whereby ART influences MTZ treatment, pharmacokinetic studies are necessary.

In summary, our secondary analyses of a previously published study support the findings of Balkus et al. in a U.S. cohort with exposure to a wider variety of HIV retroviral drugs. Since 83.2\% of our cohort was either receiving ART or had BV or both, the majority of our cohort would have been affected. These findings lend strength to the recommendation that the single $2 \mathrm{~g}$ MTZ regimen should not be used for treating HIV+ women.

\section{REFERENCES}

1. Schwebke JR, Desmond RA. A randomized controlled trial of partner notification methods for prevention of trichomoniasis in women. Sex Transm Dis. 2010; 37:392-6. [PubMed: 20453720]

2. Kissinger P, Secor WE, Leichliter JS, et al. Early repeated infections with Trichomonas vaginalis among HIV-positive and HIV-negative women. Clin Infect Dis. 2008; 46:994-9. [PubMed: 18444815]

3. Krashin JW, Koumans EH, Bradshaw-Sydnor AC, et al. Trichomonas vaginalis prevalence, incidence, risk factors and antibiotic-resistance in an adolescent population. Sex Transm Dis. 2010; 37:440-4. [PubMed: 20351623]

4. Tidwell BH, Lushbaugh WB, Laughlin MD, et al. A double-blind placebo-controlled trial of singledose intravaginal versus single-dose oral metronidazole in the treatment of trichomonal vaginitis. $\mathrm{J}$ Infect Dis. 1994; 170:242-6. [PubMed: 8014510]

5. Spence MR, Harwell TS, Davies MC, et al. The minimum single oral metronidazole dose for treating trichomoniasis: a randomized, blinded study. Obstet Gynecol. 1997; 89:699-703. [PubMed: 9166304]

6. duBouchet L, Spence MR, Rein MF, et al. Multicenter comparison of clotrimazole vaginal tablets, oral metronidazole, and vaginal suppositories containing sulfanilamide, aminacrine hydrochloride, and allantoin in the treatment of symptomatic trichomoniasis. Sex Transm Dis. 1997; 24:156-60. [PubMed: 9132982]

7. Magnus M, Clark R, Myers L, et al. Trichomonas vaginalis among HIV-Infected women: are immune status or protease inhibitor use associated with subsequent $\mathrm{T}$. vaginalis positivity? Sex Transm Dis. 2003; 30:839-43. [PubMed: 14603092]

8. Niccolai LM, Kopicko JJ, Kassie A, et al. Incidence and predictors of reinfection with Trichomonas vaginalis in HIV-infected women. Sex Transm Dis. 2000; 27:284-8. [PubMed: 10821602]

9. Kissinger P, Mena L, Levison J, et al. A randomized treatment trial: single versus 7-day dose of metronidazole for the treatment of Trichomonas vaginalis among HIV-infected women. J Acquir Immune Defic Syndr. 2010; 55:565-71. [PubMed: 21423852]

10. Gatski M, Martin DH, Levison J, et al. The influence of bacterial vaginosis on the response to Trichomonas vaginalis treatment among HIV-infected women. Sex Transm Infect. 2011; 87:2058. [PubMed: 21278401] 
Table 1

Association of ARV with Repeat TV infection at Test of Cure visit $(n=113)$

\begin{tabular}{|l|r|l|l|l|}
\hline & TOC positive & $\begin{array}{l}\text { Adjusted } \\
\text { O.R. }\end{array}$ & 95\% C.I. & p-value \\
\hline \begin{tabular}{l|l|l|l|l} 
ART \\
Yes
\end{tabular} & $23.7 \%$ & 4.58 & $1.09-19.36$ & 0.038 \\
No & $8.1 \%$ & 1.00 & & \\
\hline BV & & & & \\
Yes & $29.0 \%$ & 7.01 & $1.84-26.81$ & 0.004 \\
No & $5.9 \%$ & 1.00 & & \\
\hline CD4 & & & & \\
$<200 / \mathrm{mm}^{3}$ & $17.2 \%$ & 1.00 & - & - \\
$200-499$ & $15.0 \%$ & 0.83 & $0.23-2.99$ & 0.77 \\
2500 & $29.2 \%$ & 3.54 & $0.77-16.33$ & 0.11 \\
\hline
\end{tabular}

